\title{
Comportement de nouvelles lignées isogéniques de riz irrigué dotées du gène de résistance (rymv1) au RYMV en Afrique de l'ouest : situation en Côte d'Ivoire
}

\author{
Alphonse BOUET ${ }^{1 *}$, Acho Nicaise AMANCHO ${ }^{2}$, Nazaire KOUASSI $^{2}$ et \\ Kouamé ANGUETE ${ }^{1}$ \\ ${ }^{I}$ Centre National de Recherche Agronomique (CNRA), BP 602 Gagnoa, Côte d'Ivoire. \\ ${ }^{2}$ Laboratoire Central de Biotechnologies, Adiopodoumé, Km 17, route de Dabou, 01 BP 1740 Abidjan 01, \\ Côte d'Ivoire. \\ *Auteur correspondant, E-mail: bouetalph@yahoo.fr ; Tel : (225)10 5010 93/ 0742 64 85/ 55324343 ; \\ Tél/fax : (225)32 771700
}

\section{RESUME}

La production rizicole nationale en Côte d'Ivoire est déficitaire depuis plusieurs années pour des raisons diverses parmi lesquelles les pertes de récolte dues à la panachure jaune (RYMV), notamment en riziculture irriguée, constituent l'une des principales. Des efforts de recherche ont abouti à la sélection de deux variétés de riz résistantes (Bouaké-am et WAS63-22-5-1-7-7-RPJ) pour relayer les variétés Bouaké 189 et WITA9 plus ou moins sensibles à la maladie. Cependant, face à la diversité biologique du RYMV, la recherche d'autres génotypes de riz résistants se poursuit. Aussi, des essais ont-ils été conduits en milieux réel et contrôlé afin de sélectionner au sein de génotypes de riz les individus résistants avec en plus un bon profil agronomique. Le dispositif expérimental adopté dans l'ensemble a été un bloc de Fischer à 4 répétitions. En milieu réel, les travaux ont eu pour sites Sogbonon (Dabakala, au Centre), Kouitongouiné (Man, à l'Ouest) et Tiassalé (au Sud), des localités traditionnellement endémiques de panachure jaune. Les résultats ont révélé en général que les quatre lignées isogéniques (NIL2, NIL16, NIL54, NIL130) présentent un haut niveau de résistance face à la diversité biologique du RYMV en Côte d'Ivoire. Cette information permet d'envisager le déploiement, sans grand risque de contournement du gène rymv1-2 présent dans les lignées isogéniques. Par ailleurs, NIL130 et secondairement NIL2 se sont distinguées au niveau agronomique. Par conséquent, la variété NIL130 peut être une alternative à WITA9 et Bouaké 189 pour la riziculture irriguée en Côte d'Ivoire. Cependant, pour que cette variété exprime son potentiel agronomique, il faut entre autres un bon aménagement hydro-agricole du bassin de culture. Cela suppose, un travail de sol approprié et une maîtrise dans la gestion de l'eau d'irrigation.

(c) 2013 International Formulae Group. All rights reserved.

Mots clés: Sélection, panachure jaune, riziculture aquatique, variété, milieu semi-contrôlé, milieu réel.

\section{INTRODUCTION}

En Afrique de l'Ouest, le riz (Oryza sativa L.) constitue un aliment vital pour plusieurs pays, notamment la Côte d'Ivoire, le
Sénégal, le Mali et la Gambie. En Côte d'Ivoire, depuis plus de trente ans, après la dissolution de la Société de Développement du Riz (SODERIZ), la production rizicole 
locale est demeurée en de çà du besoin social de consommation estimé aujourd'hui à 1500 000 tonnes de riz blanchi (Anonyme 1, 2010). Le déficit de production d'environ 50\% (Anonyme 1, 2010) est comblé par des importations qui ont coûté, en 2009, 235 milliards de francs CFA, soit un peu plus de 900000 tonnes de riz blanchi.

Parmi les principales causes à la faible productivité du riz en Côte d'Ivoire, figure la panachure jaune (ou RYMV = Rice Yellow Mottle Virus) appelée localement «SIDA du riz ». La Panachure jaune est une maladie virale plus fréquente et préjudiciable en riziculture aquatique (riziculture irriguée, riziculture de bas-fond). L'ampleur des dégâts au champ et de pertes de production induits par cette virose ruinent les espoirs agronomiques et financiers des exploitants dont certains abandonnent leurs champs affectés en Côte d'Ivoire (Bouet et al., 2001 ; Vales et al., 2001 ; Amancho et al., 2008 ).

Des méthodes de lutte contre le RYMV sont disponibles. Parmi elles, la lutte génétique est non seulement efficace mais, la plus appropriée dans le cas des pays comme la Côte d'Ivoire caractérisée par un faible pouvoir financier des paysans. Deux variétés de riz irrigué améliorées et homologuées tapissent largement les bassins rizicoles en Côte d'Ivoire. Il s'agit de Bouaké 189, apparue dans les rizières depuis les années 1980. Cette variété est très prisée par les consommateurs et les producteurs pour sa bonne qualité organoleptique (bon goût), sa bonne valeur marchande et son rendement qui peut atteindre 6 t.ha $^{-1}$ en condition optimale de culture. Malheureusement, Bouaké 189 s'est révélée sensible au RYMV dans toutes les zones rizicoles où la maladie sévit (Amancho et al., 2009 ; Bouet et al., 2010). La deuxième variété, WITA9, en grande expansion depuis la fin des années 1990 s'illustre par sa relative bonne tolérance au RYMV, son cycle court (cycle semis-maturité $=105$ jours) et son haut rendement (rendement potentiel $=9 \mathrm{t}\left(\mathrm{ha}^{-1}\right)$. Mais au cours des cinq dernières années, des attaques de plus en plus sévères sont notées sur cette dernière variété citée (Amancho et al., 2009 ; Bouet et al., 2010). Aussi se pose-til à la Côte d'Ivoire, la problématique d'une alternative à Bouaké 189 et WITA9. Cette préoccupation est d'autant plus justifiée que le pays ambitionne à travers la Stratégie Nationale de Développement de la filière Riz (SNDR) de parvenir à l'autosuffisance en riz voire engranger des excédants de production à l'orée 2020 (Anonyme 2, 2012). Les travaux de Bouet et al. (2010) ont permis de sélectionner deux variétés de riz irrigué résistantes au RYMV dans plusieurs localités de la Côte d'Ivoire. Cependant, d'autres génotypes doivent être recherchés afin de contrôler la diversité pathogénique des isolats du virus.

Le Centre de Riz pour l'Afrique (Africa Rice), grâce à la sélection assistée par marqueur, a mis au point des lignées isogéniques (NIL) comportant le gène rymv12 de résistance au RYMV. Ce gène a été introgressé dans quatre variétés de riz africain prisées (Sahelika, FKR28, IR47, IR64) à partir de la variété Gigante (génitrice de résistance). Quelques unes des NIL prometteuses sont en évaluation dans plusieurs pays d'Afrique de l'ouest à travers divers financements dont celui de l'USAID acquis par le CORAF/WECARD.

Le présent article met en lumière les résultats de l'évaluation de quatre lignées isogéniques pour la résistance au RYMV en Côte d'Ivoire. La performance agronomique de ces lignées a été par ailleurs déterminée.

\section{MATERIEL ET METHODES}

Quatre essais ont été conduits; un en milieu semi-contrôlé (en serre) et trois en milieu réel. Ils sont relatifs à « l'évaluation de génotypes de riz pour la résistance à la panachure jaune en milieu semi-contrôlé » et à «l'évaluation multilocale des nouvelles lignées isogéniques de riz dotées du gène rymv1-2 de résistance au RYMV ». 
Evaluation de génotypes de riz pour la résistance à la panachure jaune en milieu semi-contrôlé

L'essai a été réalisé dans la serre d'expérimentation, sur le site de la Direction générale du CNRA à Abidjan. Le matériel végétal utilisé était constitué de quatre lignées isogéniques de riz dotées du gène rymv1-2 de résistance au RYMV. Il s'agit de NIL2 (issue de l'introgression du gène rymv1-2 dans la variété de riz FKR28 à partir de la variété de riz Gigante), NIL16 (issue de l'introgression du gène rymv1-2 dans la variété de riz Sahelika, à partir de la variété Gigante), NIL54 (issue de l'introgression du gène rymv1-2 dans la variété de riz IR47, à partir de la variété Gigante) et de NIL130 (issue de l'introgression du gène rymv1-2 dans la variété de riz IR64, à partir de la variété Gigante). En plus de ces lignées isogéniques, les variétés de riz Gigante, Bouaké 189, WITA9 et Bouaké-am ont été utilisées. La variété Gigante est le géniteur de la résistance au RYMV (rymv1-2) quand, Bouaké 189 et WITA9 sont respectivement témoins locaux de sensibilité et de résistance au RYMV. Par ailleurs Bouaké 189 et WITA9 sont les deux variétés améliorées, homologuées et largement cultivées en Côte d'Ivoire. La variété Bouaké-am quant à elle, a été sélectionnée par le CNRA comme résistante à la panachure jaune au terme de cinq années d'étude. Le matériel viral était composé de six isolats représentatifs de la diversité pathogénique du RYMV en Côte d'Ivoire.

Le dispositif expérimental adopté est un bloc de Fisher à 4 répétitions. Chacun des génotypes de riz a été semé dans 4 pots de $2 \mathrm{~L}$ à raison de 3 plants par pot. Chaque variété de riz a été inoculée par friction manuelle avec 6 isolats du RYMV. Cela correspond à 192 traitements. L'inoculation a été réalisée à 21 jours après semis (JAS). La sévérité de la panachure jaune a été notée à $14,21,28,35$, 42,49 et 60 jours après l'inoculation (JAI). L'échelle d'évaluation standard de l'IRRI (2009) a été utilisée à cet effet.
Evaluation multilocale de nouvelles lignées isogéniques de riz dotées du gène rymv1-2 de résistance au RYMV

Les travaux ont été conduits de 2010 à 2011 à Dabakala (bas-fond de Sogbonon), à Man (Ketongouiné) et à Tiassalé (sur le périmètre exploité par la coopérative CODERIZ), dans des bas-fonds rizicoles où, la panachure jaune a souvent sévi.

Le matériel végétal utilisé était constitué au total de 07 variétés de riz réparties comme suit :

quatre lignées isogéniques (NIL2, NIL16, NIL54, NIL130) dotées du gène rymv1 de résistance à la panachure jaune, mises au point et fournies par le Centre de Riz pour l'Afrique (Africa Rice) ;

la variété de riz irrigué WITA9 cultivée, (homologuée et adoptée par les riziculteurs), comme témoin de tolérance à la panachure jaune ;

la variété de riz irrigué Bouaké-am, sélectionnée par le CNRA comme résistante à la panachure jaune au terme de 5 années d'étude en Côte d'Ivoire ;

la variété Bouaké 189 (améliorée, homologuée et très cultivée), comme témoin de sensibilité à la panachure jaune.

Un dispositif en bloc de FISHER à 4 répétitions a été adopté. Le facteur étudié est le génotype de riz avec sept niveaux. Chacun des génotypes étudiés a été semé dans une parcelle élémentaire matérialisée par 6 lignes, dans des poquets alignés. Le matériel végétal a été mis en pépinière pendant 15 à 20 jours avant de le repiquer à un brin, dans la parcelle élémentaire. Les plants repiqués étaient espacés les uns des autres de 0,20 m. La parcelle élémentaire couvrait $2 \mathrm{~m}^{2}$ ( $2 \mathrm{~m}$ x 1 m). Aucun apport d'engrais n'a été assuré. Les plants ont été soumis à la pression naturelle du RYMV. Pour déclencher et homogénéiser l'épidémie dans le champ d'essai, des bandes infestantes, emblavées avec la variété sensible Bouaké 189 ont été installées entre et autour des blocs

Les notations scientifiques au champ ont porté sur : 
- Le nombre de plantes (talles principales) attaquées par la panachure jaune à 70 JAR (jours après repiquage) ;

- le nombre total de talles;

- le nombre de talles fertiles (nombre de panicules par plante) ;

- le cycle semis-50\% épiaison ;

- la hauteur des plantes ;

- le rendement (t/ha) en grains.

Les analyses statistiques ont été effectuées par le logiciel GENSTAT.

\section{RESULTATS}

Evaluation phytopathologique de génotypes de riz pour la résistance à la panachure jaune

Réaction des variétés de riz au virus de la panachure jaune

En milieu semi-contrôlé, l'analyse statistique des notes enregistrées révèle un effet significatif du génotype sur la sévérité des symptômes du RYMV aux 14, 21, 28 et 60 jours après inoculation (JAI). Parmi les variétés, NIL16, NIL54 et NIL130 sont restées indemnes de symptôme de RYMV quand, les autres (NIL2, Bouaké-am, WITA9, Bouaké 189) présentaient des symptômes plus ou moins apparents sur les feuilles (Tableau 1). Dans ce dernier groupe variétal cité, une mention spéciale est faite à la variété Bouaké 189 qui affiche des notes de sévérité relativement élevées, proches de 4 aux 21, 28 et 60 JAI. Cependant, les réactions des variétés au RYMV sont dans l'ensemble, celles de résistance pour NIL2, NIL16, NIL54, NIL130, WITA9 et Bouaké-am. Celles-ci ont en effet obtenu des notes de sévérité inférieures à 3 (Tableau 1). Ce résultat a été confirmé par les travaux conduits en milieu réel en 2011 à Tiassalé (Tableau 4). En 2010, l'épidémie de RYMV a été inexistante aussi bien à Sogbonon (Dabakala) qu'à Kétongouiné (Man). Il en a été de même dans les rizières paysannes environnantes.
Développement des symptômes de la panachure jaune

L'observation de la Figure 1 relative à l'évolution de la sévérité des symptômes du RYMV en fonction des dates de notation révèle que les premiers symptômes étaient

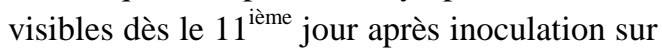
les variétés Bouaké 189 et WITA9. Sur les variétés NIL2 et Bouaké-am, les premiers symptômes ont été observés au $14^{\text {ième jour }}$ après inoculation. Dans l'ensemble, l'intensité des symptômes du RYMV augmente et atteint son optimum entre le $11^{\text {ième }}$ et le $14^{\text {ième }}$ jour après inoculation. Au-delà du 28 ième jour après inoculation, la sévérité du RYMV reste stationnaire.

\section{Pouvoir pathogène des isolats viraux utilisés}

Les Figures 2 à 8 montrent, par variété de riz testé, la note moyenne de sévérité des symptômes après inoculation avec les isolats CI24, CI39, CI97, CI169 et CI188 du RYMV. Il ressort deux constats. Le premier est qu'aucun des isolats n'a provoqué de symptôme sur les variétés NIL16, NIL54 et NIL130. Le deuxième porte sur les isolats CI97, CI169 et CI188, relativement virulents sur les variétés Bouaké 189 (note de sévérité = 5), WITA9 (note de sévérité = 4), Bouaké-am (note de sévérité $=3$ ) et NIL2 (note de sévérité $=2$ ).

\section{Evaluation agronomique des variétés testées \\ Tallage}

Les analyses de variance effectuées à travers la variable «talles totales » ont révélé une différence significative entre les variétés de riz étudiées aussi bien à Sogbonon qu'à kouitongouiné (Tableaux 1 et 2).

A Sogbonon, la variété la plus productive est NIL130 avec une note de 4 talles émises par plante. Avec les nombres de talles compris entre 1 et 2,5, les variétés Giganté, IR64, FKR28, NIL2, Sahelika, 
NIL16, WITA9, Bouaké 189 et Bouaké-am s'affichent comme moyennement productives en talles. Les plus faibles notes ayant été acquises par les variétés IR47 et NIL54. Il faut remarquer par ailleurs que seule la variété NIL130 a été meilleure que les témoins Bouaké 189 et WITA9 (Tableau 1).

Concernant le site de Kouitongouiné, 4 groupes variétaux distincts ont été révélés par l'analyse statistique (Tableau 2). Le groupe composé de NIL130 et Bouaké 189 vient en tête avec les plus grands nombres de talles émises. Il est suivi du singleton représenté par la variété Bouaké-am avec plus de 9 talles produites. Le troisième groupe variétal comprend NIL16, NIL2 et WITA9 avec, dans l'ordre, les notes de 7,94, 8,44 et 7,06 talles. La variété NIL54 constitue le dernier groupe. La note (5,44 talles) acquise par cette dernière variété est au moins deux fois inférieure à celles obtenues par Bouaké 189 et NIL130.

Comme à Sogbonon et à Kouitongouiné, l'analyse statistique a montré un effet significatif de la variété de riz sur le nombre de talles totales émises à Tiassalé. Selon cette variable analysée, les variétés NIL130 et WITA9 ont affiché les valeurs les plus élevées avec respectivement 16 et 15 talles (Tableau 5).

En considérant le nombre de talles fertiles produites par plante, l'analyse statistique a mis en évidence des différences significatives entre génotypes de riz dans les localités de Sogbonon et de Tiassalé. Ainsi, à Sogbonon, les variétés Giganté et NIL130 ont eu les nombres de talles les plus élevés, compris entre 2,6 et 3,5. A leur suite, se distinguent les variétés NIL2, Bouaké 189 et Bouaké-am avec des notes de 2,20, 1,900 et 2,3 talles respectivement. Les autres variétés, notamment NIL16 (1,2 talles) et WITA9 (1,8 talles) s'étant illustrées par des nombres faibles. La variété NIL54 présente le plus faible nombre de talles fertiles produites. A Tiassalé, les variétés NIL130 et WITA9 se sont illustrées comme les plus prolifiques avec les nombres de 14 et de 15 talles respectivement.
Sur le site de Kouitongouiné, bien que toutes les variétés se valent statistiquement, le groupe constitué par NIL130, Bouaké 189 et NIL2 apparaît comme le plus productif en valeur absolue. La variété NIL54 demeurant toujours la moins productive.

\section{Hauteur des plantes}

L'analyse statistique des données relatives à la hauteur des plantes montre un effet significatif du génotype de riz à Sogbonon, kouitongouiné et à Tiassalé.

Concernant Sogbonon, quatre groupes de variétés ont été mis en évidence (Tableau 2). Il y a en premier, l'ensemble constitué par Bouaké-am, Bouaké 189, NIL54, IR47, Shahelika, FKR28 et Giganté qui présente les hauteurs les plus élevées, comprises entre 79 $\mathrm{cm}$ et $86 \mathrm{~cm}$. Le deuxième est composé par NIL16 et NIL130 avec des hauteurs respectives de $75,70 \mathrm{~cm}$ et $74,50 \mathrm{~cm}$. Les deux premiers groupes cités peuvent être qualifiés, de « haute taille». Les deux derniers groupes représentés par, d'une part, NIL2 $(71,85 \mathrm{~cm})$ et IR64 $(72,95 \mathrm{~cm})$ et, d'autre part, WITA9 $(67,70 \mathrm{~cm})$ peuvent être considérés comme de « taille courte .

A Kouitongouiné, trois catégories de taille s'affichent (Tableau 3). La première regroupe les variétés Bouaké-am, Bouaké 189, NIL54 dont les hauteurs sont comprises entre $92 \mathrm{~cm}$ et $96,5 \mathrm{~cm}$. La catégorie intermédiaire est représentée par NIL2 avec une hauteur de $78,5 \mathrm{~cm}$. Les variétés NIL16, WITA9 et NIL130 appartiennent à la dernière catégorie, avec des hauteurs variant de $73 \mathrm{~cm}$ à $78,01 \mathrm{~cm}$ (Tableau 3).

Sur le site de Tiassalé, deux groupes de tailles sont révélés par l'analyse statistique (Tableau 4). Le premier comporte les variétés NIL54 (116 cm), Bouaké $189(104 \mathrm{~cm})$, Bouaké-am $(108 \mathrm{~cm})$ et NIL16 $(96 \mathrm{~cm})$ avec des nombres de talles compris entre 96 et 117 $\mathrm{cm}$. Le second groupe est constitué par les variétés NIL2 $(89,95 \mathrm{~cm})$, WITA9 $(85,55 \mathrm{~cm})$ et NIL130 $(87,47 \mathrm{~cm})$ avec des hauteurs inférieures à $90 \mathrm{~cm}$. 


\section{Cycle semis- 50\% épiaison des variétés}

Selon l'analyse statistique, le génotype de riz n'a eu aucun effet significatif sur le cycle semis-50\% épiaison à Sogbonon et à Kouitongouiné, contrairement à Tiassalé (Tableau 4). Notons cependant, qu'en valeur absolue, la variété NIL54 a eu le plus court cycle, avec NIL130 et WITA9 dans les deux premières localités citées (Tableaux 2 et 3). Concernant Tiassalé, trois groupes variétaux se distinguent. Un groupe renfermant les variétés WITA9 et NIL130 de cycle très court (92 jours). Un autre groupe avec les variétés NIL2 et NIL54 de cycle court, compris entre 96 et 99 jours. Enfin, le groupe constitué par Bouaké 189, Bouaké-am et NIL16 de cycle moyen, situé entre 101 et 105 jours (Tableau 4).

\section{Rendement en grain des variétés}

Les analyses statistiques ont montré un effet significatif du génotype de riz sur le rendement à Sogbonon et à Kouitongouiné (Tableaux 2 et 3 ).

Le constat général sur le site de Sogbonon est que tous les rendements obtenus sont inférieurs à 1 t.ha ${ }^{-1}$ (Tableau 2). Cependant, trois groupes variétaux se dégagent. Le groupe le plus productif relativement, renferme les variétés NIL2 et Gigante, avec des rendements respectifs de 0,416 t.ha $^{-1}$ et 0,346 t.ha $^{-1}$. Le deuxième ensemble formé par les variétés NIL130-am, NIL16 et Bouaké 189 a affiché des rendements moyens compris entre 0,215 tha ${ }^{1}$ et 0,268 t.ha $^{-1}$. Les variétés IR64, Sahelika, FKR28, Bouaké-am et WITA9, constituant le dernier groupe, avec des rendements très faibles (inférieurs à 0,2 t.ha" ${ }^{-1}$ ).

Concernant Kouitongouiné, quatre ensembles variétaux se distinguent (Tableau 3). Le premier est formé par Bouaké-am, Bouaké 189 et NIL2 avec des rendements respectifs de 3,01 t.ha ${ }^{-1}, 3,52$ t.ha $^{-1}$ et 4,31 t.ha 1 (Tableau 3). Le deuxième ensemble regroupe les variétés NIL130 et NIL16 qui ont acquis dans l'ordre, des rendements moyens de 2,16 tha ${ }^{-1}$ et 2,69 t.ha ${ }^{-1}$. Les variétés
NIL54 et WITA9, avec des rendements inférieurs à $2 \mathrm{t}$ ha 1 , constituent le groupe le moins productif du matériel végétal testé. Remarquons que les variétés NIL2, NIL130 et NIL16 se sont révélées plus productives que WITA9, un des témoins utilisés. Concernant l'autre témoin Bouaké 189, il a affiché une performance agronomique supérieure à celles des NILs, excepté NIL2, la plus productive en valeur absolue (Tableau 3).

A Tiassalé, les variétés WITA9 et NIL130 semblent être les plus productives bien que l'effet variétal n'a pas été significatif sur le rendement (Tableau 4). Les variétés NIL2 et NIL54 avec des rendements moyens de 5 t.ha ${ }^{-1}$ peuvent constituer un second.

Il faut cependant remarquer dans l'ensemble, que les rendements obtenus à Tiassalé sont au moins 1,5 fois supérieurs à la moyenne nationale qui est de 3 t.ha $^{-1}$ en riziculture irriguée.

\section{Performance général du matériel végétal étudié}

En considérant, d'une part, toutes les variables analysées et d'autre part, les spécificités de la riziculture irriguée et les préoccupations des riziculteurs (taille courte à moyenne, prévalence du RYMV, haut rendement, cycle court à moyen), les variétés NIL130,t WITA9 et Bouaké-am présentent les meilleurs profils. A un degré moindre, NIL16 peut rejoindre cette élite. Dans le cas spécifique de NIL130, les données scientifiques affichées par cette variété corroborent et justifient les choix opérés par quelques acteurs (riziculteurs, transformateurs, agents de l'Etat: ANADER, Ministère de l'agriculture) de la filière riz qui ont visité les parcelles à Gagnoa et à Tiassalé. Contrairement aux autres NIL étudiées, la variété NIL54 n'est pas performante en riziculture irriguée. Cela étant dû sûrement à ses caractéristiques qui a priori la rattachent aux variétés de riz de type pluvial. Il sera intéressant de l'évaluer dans cette écologie pluviale. 
Tableau 1: Sévérité des symptômes du RYMV de 14 JAI à 60 JAI.

\begin{tabular}{lcccc}
\hline Variétés & \multicolumn{4}{c}{ Moyenne de sévérité des symptômes } \\
\cline { 2 - 5 } & 14 JAI & 21 JAI & 28 JAI & 60 JAI \\
\hline NIL 16 & $1 \mathrm{a}$ & $1 \mathrm{a}$ & $1 \mathrm{a}$ & $1 \mathrm{a}$ \\
NIL54 & $1 \mathrm{a}$ & $1 \mathrm{a}$ & $1 \mathrm{a}$ & $1 \mathrm{a}$ \\
NIL130 & $1 \mathrm{a}$ & $1 \mathrm{a}$ & $1 \mathrm{a}$ & $1 \mathrm{a}$ \\
NIL2 & $1 \mathrm{a}$ & $1,25 \mathrm{a}$ & $1,50 \mathrm{~b}$ & $1,41 \mathrm{a}$ \\
Bouaké amélioré & $1,14 \mathrm{a}$ & $1,44 \mathrm{a}$ & $1,44 \mathrm{ab}$ & $1,44 \mathrm{ab}$ \\
WITA 9 & $1,44 \mathrm{a}$ & $2,08 \mathrm{~b}$ & $2,16 \mathrm{c}$ & $2,16 \mathrm{~b}$ \\
Bouaké 189 & $1,94 \mathrm{~b}$ & $3,33 \mathrm{c}$ & $3,33 \mathrm{~cd}$ & $3,33 \mathrm{c}$ \\
Signification & HS & HS & HS & HS \\
\hline
\end{tabular}

Tableau 2: Moyennes des paramètres agronomiques et phénologiques, notés sur les variétés étudiées à Sogbonon (Dabakala).

\begin{tabular}{lcccc}
\hline Variétés & $\begin{array}{c}\text { Talles } \\
\text { totales }\end{array}$ & $\begin{array}{c}\text { Nombre de } \\
\text { panicules par plante }\end{array}$ & $\begin{array}{c}\text { Hauteur de la } \\
\text { plante (cm) }\end{array}$ & $\begin{array}{c}\text { Rendement } \\
\text { grains (t/ha) }\end{array}$ \\
\hline Gigante & 2.500 & 2.650 & 85.65 & 0.346 \\
IR-64 & 1.950 & 1.750 & 72.95 & 0.151 \\
NIL130 & 4.00 & 3.400 & 74.50 & 0.268 \\
FKR28 & 2.00 & 1.800 & 80.95 & 0.159 \\
NIL2 & 2.30 & 2.200 & 71.85 & 0.416 \\
Sahelika & 2.20 & 1.700 & 81.15 & 0.195 \\
NIL16 & 2.40 & 1.700 & 75.70 & 0.226 \\
IR47 & 1.30 & 1.200 & 81.25 & 0.118 \\
NIL54 & 1.05 & 1.050 & 79.60 & 0.115 \\
WITA9 & 1.650 & 1.650 & 67.70 & 0.181 \\
Bouaké 189 & 2.10 & 1.900 & 79.95 & 0.215 \\
Bouaké-am & 1.950 & 2.300 & 86.15 & 0.235 \\
Moyenne générale & 2.154 & 1.942 & 78.12 & 0.219 \\
Probabilité & $<0.001$ & $<0.001$ & $<0.001$ & 0.004 \\
Ppds (lsd) & 0.9856 & 0.7531 & 6.611 & 0.143 \\
Cv (\%) & 12.5 & 9.5 & 2.3 & 26.5 \\
\hline
\end{tabular}


Tableau 3: Moyennes des variables agronomiques et phénologiques, notées sur les variétés de riz à Kouitongouiné (Man).

\begin{tabular}{lccccc}
\hline Variétés & $\begin{array}{c}\text { Talles } \\
\text { totales }\end{array}$ & $\begin{array}{c}\text { Nombre de } \\
\text { panicules par } \\
\text { plante }\end{array}$ & $\begin{array}{c}\text { Hauteur de la } \\
\text { plante }(\mathbf{c m})\end{array}$ & $\begin{array}{c}\text { Cycle semis- } \\
\mathbf{5 0 \%} \text { épiaison } \\
\text { (jours) }\end{array}$ & $\begin{array}{c}\text { Rendement } \\
\text { grains } \\
\text { (t/ha) }\end{array}$ \\
\hline NIL130 & 12.56 & 7.94 & 73.19 & 99.00 & 2.16 \\
NIL2 & 8.44 & 6.50 & 78.56 & 100.00 & 4.31 \\
NIL16 & 7.94 & 6.06 & 78.00 & 103.00 & 2.69 \\
NIL54 & 5.44 & 3.75 & 96.06 & 97.50 & 0.73 \\
WITA9 & 7.06 & 5.38 & 75.12 & 99.00 & 1.34 \\
Bouaké 189 & 11.56 & 7.06 & 92.94 & 105.00 & 3.52 \\
Bouaké-am & 9.19 & 5.94 & 96.44 & 100.5 & 3.01 \\
Moyenne générale & 8.88 & 6.09 & 84.33 & 100.57 & 2.54 \\
Probabilité & $<0.001$ & 0.005 & $<0.001$ & 0.089 & 0.001 \\
Ppds (lsd) & 2.764 & 1.804 & 7.289 & 5.163 & 1.515 \\
Cv (\%) & 1.3 & 5.50 & 4.9 & 0.5 & 29.60 \\
\hline
\end{tabular}

Tableau 4: valeurs moyennes acquises par les variétés à Tiassalé, selon les variables analysés.

\begin{tabular}{lcccccc}
\hline Variété de riz & $\begin{array}{c}\text { RYMV } \\
\text { (\%plantes } \\
\text { attaquées) }\end{array}$ & $\begin{array}{c}\text { Cycle semis- } \\
\text { 50\%épiaison } \\
\text { (jours) }\end{array}$ & $\begin{array}{c}\text { Rendement } \\
\text { paddy } \\
\text { (t/ha) }\end{array}$ & $\begin{array}{c}\text { Nombre } \\
\text { de talles } \\
\text { fertiles }\end{array}$ & $\begin{array}{c}\text { Nombre } \\
\text { de talles } \\
\text { totales }\end{array}$ & $\begin{array}{c}\text { Hauteur } \\
\text { (cm) }\end{array}$ \\
\hline NIL2 & $0 \mathrm{~b}$ & $97 \mathrm{~b}$ & 5,25 & $13,58 \mathrm{a}$ & $14,52 \mathrm{a}$ & $89,95 \mathrm{a}$ \\
NIL16 & $0 \mathrm{~b}$ & $105 \mathrm{a}$ & 4,92 & $11,28 \mathrm{~b}$ & $12,95 \mathrm{~b}$ & $96,62 \mathrm{~b}$ \\
NIL54 & $0 \mathrm{~b}$ & $98,25 \mathrm{~b}$ & 5,17 & $8,68 \mathrm{c}$ & $9,30 \mathrm{c}$ & $116,92 \mathrm{~b}$ \\
NIL130 & $0 \mathrm{~b}$ & $92 \mathrm{c}$ & 6,67 & $14,28 \mathrm{a}$ & $16,22 \mathrm{a}$ & $87,47 \mathrm{a}$ \\
Bouaké 189 & $5,72 \mathrm{a}$ & $104 \mathrm{a}$ & 4,92 & $11,30 \mathrm{~b}$ & $12,42 \mathrm{~b}$ & $104,00 \mathrm{~b}$ \\
Bouaké-am & $0 \mathrm{~b}$ & $102,75 \mathrm{a}$ & 4,50 & $11,90 \mathrm{~b}$ & $12,95 \mathrm{~b}$ & $108,88 \mathrm{~b}$ \\
WITA9 & $0 \mathrm{~b}$ & $92 \mathrm{c}$ & 7,34 & $15 \mathrm{a}$ & $15,50 \mathrm{a}$ & $85,55 \mathrm{a}$ \\
Moyenne & 0,82 & 98,79 & 5,54 & 12,29 & 13,41 & 98,49 \\
Probabilité (p) & $<0,001$ & $<0,001$ & 0,131 & 0,001 & $<0,001$ & $<0,001$ \\
Ppds & 0,0147 & 3,463 & 2,238 & 2,564 & 1,940 & 4,329 \\
CV (\%) & 45,8 & 0,5 & 7,4 & 6,1 & 4,9 & 3,0 \\
Effet & $\mathrm{Hs}$ & $\mathrm{hs}$ & $\mathrm{ns}$ & $\mathrm{s}$ & $\mathrm{hs}$ & $\mathrm{hs}$ \\
\hline
\end{tabular}

Effet significatif si $\mathrm{p}<0,05$. 
Note de sévérité des symptômes du RYMV

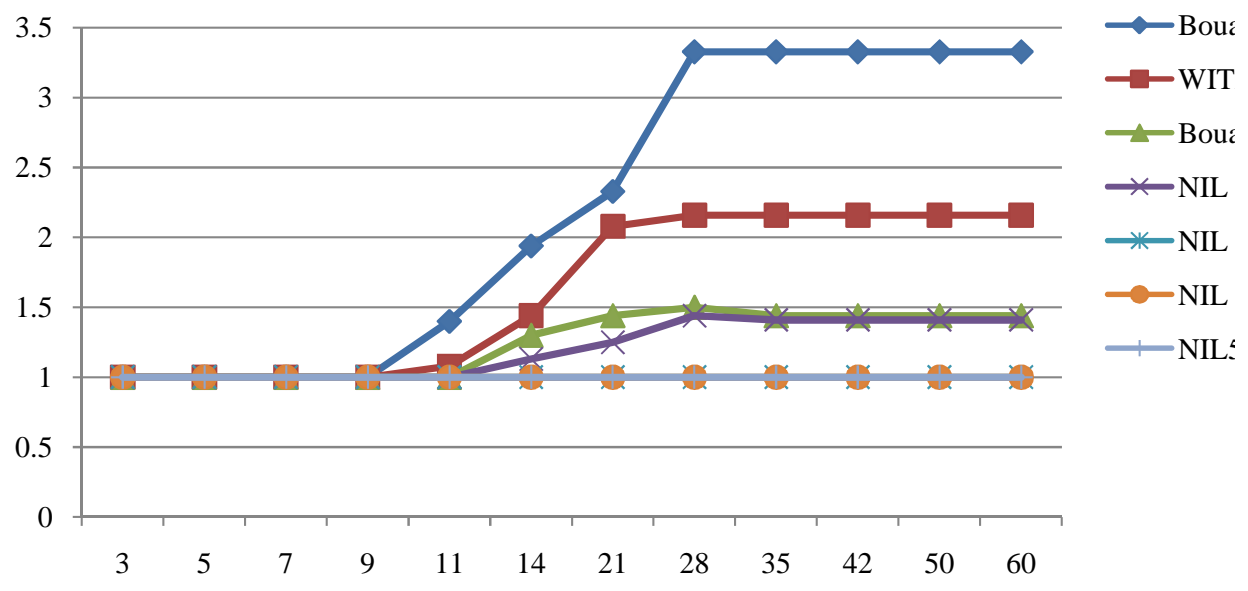

Figure 1 : Evolution de la sévérité des symptômes du RYMV sur les variétés de riz NIL2, NIL16, NIL54 et NIL130, en fonction de la date de notation (en jours après inoculation).

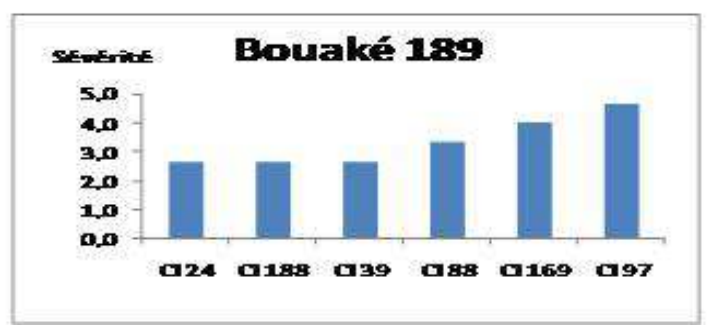

Figure 2: Sévérité des symptômes du RYMV sur la variété Bouaké 189 inoculée avec six isolats viraux.

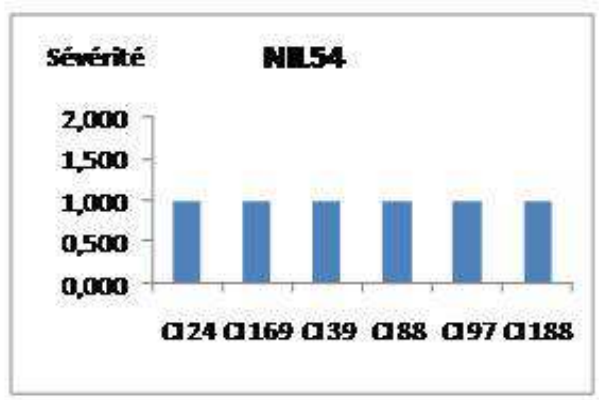

Figure 4: Sévérité des symptômes du RYMV sur la variété NIL54 inoculée avec six isolats viraux

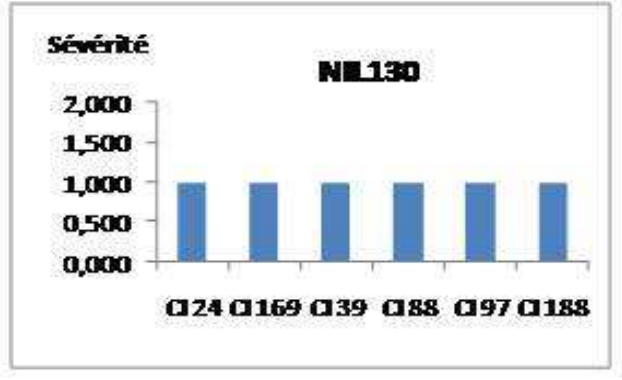

Figure 3 : Sévérité des symptômes du RYMV sur la variété NIL130 inoculée avec six isolats viraux

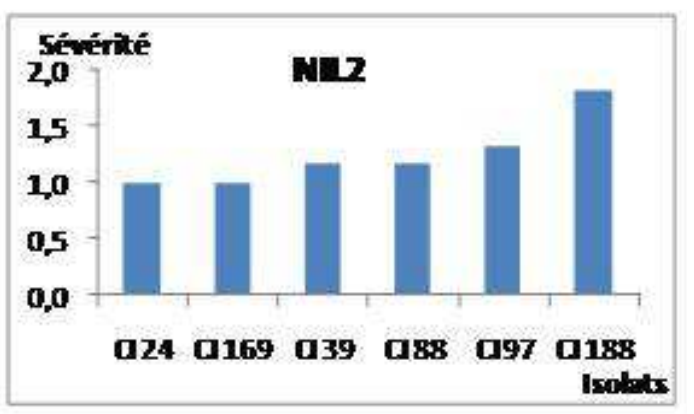

Figure 5 : Sévérité des symptômes du RYMV sur la variété NIL2 inoculée avec six isolats viraux 


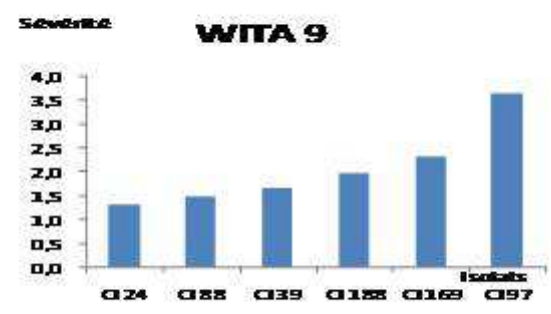

Figure 6 : Sévérité des symptômes du RYMV sur la variété WITA9 inoculée avec six isolats viraux

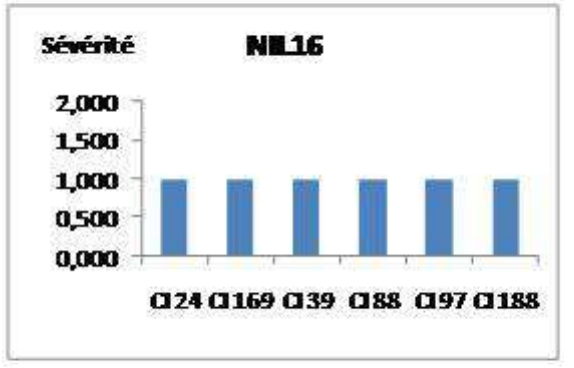

Figure 7 : Sévérité des symptômes du RYMV sur la variété NIL16 inoculée avec six isolats viraux

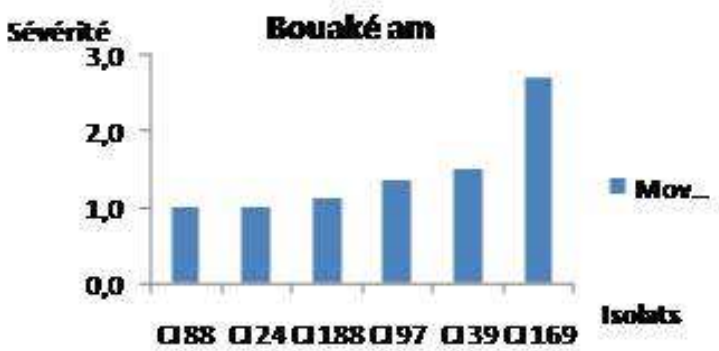

Figure 8 : sévérité des symptômes du RYMV sur la variété NIL16 inoculée avec six isolats viraux.

\section{DISCUSSION}

Le constat fait sur les sites d'essai en 2010 à Sogbonon (Dabakala) et à Kouitongouiné (Man) a été l'absence totale de maladie du RYMV. Il n'a donc pas été possible de vérifier la résistance au RYMV du matériel végétal testé, notamment les variétés NILs (NIL2, NIL16, NIL54, NIL130) dotées du gène rymv1, de résistance à la panachure jaune. La situation épidémique observée sur le site d'essai de Kouitongouiné, trouve son explication dans la variation saisonnière de la pression du RYMV. En effet, il a été montré que la dynamique de population des insectes vecteurs du RYMV fluctue dans le temps (henrisch, 2001). Rappelons que ce site a été choisi à cause de la très forte pression de RYMV qui y régnait de 2007 à 2009. Concernant Sogbonon, le RYMV ne semble pas être la cause des grandes pertes de production et de jaunissement rapportés les années antérieures par les agents de développement de l'ANADER (Agence National de Développement Rural) et de l'ONDR (Office National de Développement de la filière Riz), ex-PNR (Programme National Riz). Il a été constaté sur place, l'existence d'un complexe de contraintes telluriques. Il s'agit de la nature très argileuse et profonde (profondeur $>25 \mathrm{~cm}$ ) du sol, couplée avec les dégâts des insectes s'y abritant. Les plantes présentent en effet un aspect chétif et un mauvais tallage. Certaines plantes se dessèchent et meurent. Les faibles notes acquises relativement aux composantes de rendement (tallage, rendement grains) à Sogbonon sont probablement dues à la conjugaison des stress biotiques et abiotiques décrits plus haut.

La variation temporelle de la pression épidémique du RYMV peut empêcher une bonne évaluation phytopathologique $\mathrm{du}$ matériel végétal comme ce fut le cas à Kouitongouiné. Pour s'affranchir de cette 
contrainte environnementale et gagner par ailleurs du temps de travail, une évaluation en condition contrôlée ou semi-contrôlée est opportune. En effet la contrainte «existence de maladie » n'est pas un facteur limitant dans un environnement maîtrisé. Ainsi, les génotypes de riz dont les réactions à la panachure jaune n'ont pu être appréciées en milieu réel l'ont été en milieu semi-contrôlé. Cependant, en milieu réel, non seulement il a été possible de jauger, dans une localité, le niveau de résistance des variétés de riz à la panachure jaune mais aussi, des données fiables sur la performance agronomique de ces variétés ont été acquises.

$\mathrm{Au}$ niveau de la pathologie, les travaux ont montré comme ceux de Bouet et al. (2001) que la bonne date de notation de la sévérité du RYMV se situe au $14^{\text {ième }}$ jours après inoculation en milieu contrôlé. Cette date de notation étant différente selon les génotypes, peut être éventuellement utilisée en tant que paramètre de mesure de la résistance partielle au RYMV comme Roumen (1993) l'a montré concernant la pyriculariose foliaire du riz.

Par ailleurs, il a été noté un faible pouvoir pathogène des isolats du RYMV sur les génotypes de riz. Cependant, une tendance à l'interaction spécifique de certains isolats avec des variétés de riz est apparue. Cela est vérifié avec les patho-systèmes CI188-NIL2, CI97-WITA9, CI169-Bouaké-am et CI97Bouaké 189, bien que les réactions variétales ont été celles de résistance excepté chez Bouaké 189 et à degré moindre chez WITA9. Ce résultat laisse apparaître un risque de contournement de la résistance chez WITA9 en Côte d'Ivoire. Déjà, des travaux antérieurs ont rapporté des attaques plus ou moins sévères du virus de la panachure jaune sur WITA9 dans certaines localités du pays (Amancho et al., 2009 ; Bouet et al., 2010).

Concernant la réaction de l'ensemble des variétés étudiées au RYMV, il ressort que tous les NILs (NIL2, NIL16, NIL54, NIL130) ont affiché globalement une résistance aussi bien en milieu contrôlé qu'en milieu réel. Par conséquence, le gène rymv1-2 de résistance au RYMV peut à priori être déployé sans grand risque de contournement en Côte
d'Ivoire, contrairement au Burkina Faso (Anonyme 3, 2010). Les situations dans ces deux pays voisins paraissent incompréhensibles en considérant les sérogroupes et groupes moléculaires de RYMV existants. En effet, des cinq types moléculaires identifiés, les isolats $\mathrm{S} 1$ et $\mathrm{S} 2$ sont exclusivement présents en Côte d'Ivoire, au Burkina Faso, au Mali, Ghana et au Nigeria quand, l'isolat S3 est le seul et spécifique de la Sierra Leone. Les groupes S4 et S5 étant inféodés à l'Afrique de l'Est (Kouassi et al., 2005). Mais la situation est plus probable si l'explication s'appui sur la diversité pathogénique (pathotypes) au sein des entités biochimiques et moléculaires $\mathrm{S} 1$ et S2. Ce cas de figure a été rapporté en Côte d'Ivoire par Bouet et al. (2006) concernant le champignon responsable de la pyriculariose.

Sur le plan agronomique, des informations utiles ont été acquises sur le matériel végétal étudié. Dans les situations difficiles comme à Sogbonon, marqué par un sol inapproprié et des inondations fréquentes, les variétés de riz n'ont pu exprimer leur potentiel agronomique. Cependant, la variété NIL2 a pu s'illustrer, bien que le rendement obtenu soit 7,5 fois plus faible que la moyenne nationale $\left(3\right.$ t.ha $\left.^{-1}\right)$ en riziculture irriguée. La variété Giganté qui partage le même groupe de rendement que NIL2 étant un cultivar traditionnel rustique adapté à la riziculture pluviale au Mozambique.

Dans le cas de Kouitongouiné, les variétés de riz se sont mieux exprimées avec des notes des variables mesurées, 2 à 5 fois supérieures à celles enregistrées à Sogbonon. Les raisons principales à ces bonnes performances sont la nature du sol appropriée, un suivi normal de l'essai et un drainage des parcelles relativement bon. En considérant le rendement, le cycle et la taille, les meilleures variétés sont dans l'ordre NIL130 et NIL2. Les variétés NIL16 et NIL54 n'ayant pas mieux fait que les témoins locaux Bouaké189 et WITA9, homologuées et largement cultivées en Côte d'Ivoire.

A Tiassalé, où la gestion d'eau d'irrigation était bien maîtrisée, les variétés de riz se sont d'avantage mieux comportées, 
comparativement à Sogbonon et Kouitongouiné. Dans l'ensemble, NIL130, WITA9 et NIL2 ont présenté les meilleurs profils agronomiques.

Le comportement agronomique du matériel végétal étudié sur les trois sites d'essai traduit une certaine sensibilité au niveau d'aménagement et à la gestion de l'eau des bassins de culture. En effet, du bas-fond sommairement aménagé, sans maîtrise d'eau de Sogbonon, en passant par le périmètre sommairement aménagé avec un bon drainage de Kouitongouiné, à Tiassalé où le bassin rizicole est aménagé avec une maîtrise de la gestion d'eau, les rendements augmentent pour atteindre leur maximum. Il faut faire remarquer que les données relatives aux composantes de rendement et phénologiques, acquises à Sogbonon, Kouitongouiné et à Tiassalé, l'ont été en condition «sans fertilisation ». Avec un apport normal d'engrais, les résultats seront optimaux, notamment pour les variétés NIL130, NIL2 et Bouaké-am.

De toutes les variétés NILs étudiées, NIL54 n'a pas été citée dans les préférences agronomiques dans le cadre de la présente étude. Pour cause, les caractéristiques phénologiques (taille) et agronomiques (tallage et rendement), de cette variété la rendent inappropriée pour une riziculture irriguée performante dont a besoin la Côte d'Ivoire. La seule satisfaction avec cette variété qui présente par ailleurs une architecture de riz japonica, est son cycle très court (très précoce). Peut être qu'il faudra la tester en condition de riziculture pluviale (basfond pluvial, milieu exondé). Ce type de matériel peut en effet être une réponse aux contraintes liées au changement climatique, notamment la rareté et l'insuffisance de pluie.

\section{Conclusion}

Les travaux conduits aussi bien au champ qu'en milieu semi-contrôlé ont montré que les nouvelles variétés isogéniques (NIL2, NIL16, NIL54, NIL130) dotées du gène rymv1-2 de résistance au RYMV peuvent être déployées sans grand risque de contournement en Côte d'Ivoire. Cependant, ces variétés ne paraissent pas toutes adaptées à la riziculture irriguée. La variété NIL54 en est l'illustration bien qu'elle soit parmi les plus précoces. Au niveau agronomique, NIL130 et à degré moindre NIL2 ont présenté de bons profils. Ces deux dernières variétés, notamment NIL130 peuvent être une alternative à WITA9 et Bouaké 189 pour la riziculture irriguée en Côte d'Ivoire. Cependant, pour que ces variétés NIls irriguées expriment leur potentiel agronomique, il faut entre autres un bon aménagement hydro-agricole du bassin de culture. Cela suppose, un travail de sol approprié et une maîtrise dans la gestion de l'eau d'irrigation.

\section{REMERCIEMENTS}

Le matériel végétal utilisé dans les essais, notamment les lignées isogéniques (NIL2, NIL16, NIL54, NIL130) et les variétés témoins (Gigante, Sahelika, IR54, IR60) ont été mises au point et/ou fournies par le Centre de Riz pour l'Afrique (Africa Rice). Nous adressons toute notre reconnaissance à cette institution panafricaine. Par ailleurs, les travaux rapportés dans cette publication ont été réalisés dans le cadre du projet CORAF/RYMV $\left(\mathrm{N}^{\circ} \mathrm{CW} / 03 / 01 \mathrm{CM} / \mathrm{U} / 09, \ldots\right.$. Financé par l'Agence Internationale de Développement des Etats Unis d'Amérique (USAID), via le CORAF/WECARD. Que ces deux organisations de développement et de recherche trouvent ici l'expression de toute notre gratitude.

Nous voulons enfin adresser une mention spéciale à Feu AMANCHO Acho Nicaise, jeune étudiant, bon chercheur en devenir, décédé en avril 2012 à la tâche. Feu AMANCHO Acho Nicaise, alors qu'il avait terminé ses travaux de thèse de doctorat unique et qu'il était en instance de soutenance, a conduit l'essai relatif à l'Evaluation de génotypes de riz pour la résistance à la panachure jaune en milieu semi-contrôlé. Nous rendons hommage à cet étudiant dont la sobriété cache mal le volume et la bonne qualité des travaux de recherche réalisés. Paix à son âme. 


\section{REFERENCES}

Amancho AN, Diallo HA, Kouassi KN, Bouet A, N'guessan PK. 2009. Criblage de quelques variétés de riz de Côte d'Ivoire pour la résistance à la panachure jaune du riz : incidence de la maladie sur quelques caractères agronomiques. Sciences \& Nature, 6(1): 27-37.

Amancho AN, Kouassi KN, Diallo AH, Bouet A, Aîdara D, Sangaré A. 2008. Etude épidémiologique de la panachure jaune du riz : Distribution et incidence sur les variétés de riz (Oryza sativa) cultivées en Côte Ivoire. Agronomie Africaine, 20: 201-211.

Anonyme 1. 2010. Programme de Productivité Agricole en Afrique de l'Ouest volet riz (PPAAO-1C); Projet de document de formulation de la Côte d'Ivoire. Ministère de l'Agriculture, Programme National Riz, République de Côte d'Ivoire, 36 p.

Anonyme 2. 2012. Stratégie nationale révisée de développement de la filière riz en Côte d'Ivoire (SNDR) 2012-2020. Office National de Développement de la Riziculture (ONDR), Ministère de l'Agriculture de Côte d'Ivoire, 40 p.

Anonyme 3. 2010. Rapport annuel du projet CORAF/WECARD $\quad n^{\circ} \quad$ CW/03/01

$\mathrm{CM} / \mathrm{U} / 09$, intitulé Evaluation et déploiement de variétés dotées du gène rymv1-2, résistant à la panachure jaune en Afrique de l'Ouest. INERA, Burkina Faso, 08 p.

Bouet A, Amancho NA. 2010. Sélection de deux nouvelles variétés de riz irrigué (Oryza sativa L.) à un haut niveau de résistance au virus de la panachure jaune
(RYMV) en Côte d'Ivoire. Agronomie Africaine, 22(3): 285-293.

Bouet A, Yoboue N, Vales M. 2001. Méthode d'inoculation, pour le criblage variétal de la résistance du riz (Oryza sativa L.) à la panachure jaune ou RYMV (Rice Yellow Mottle Virus). In La panachure jaune du riz : Importance économique, diagnostic et stratégie de gestion Abdoul Aziz Sy, Jacqueline Hughes, Alassane Diallo, (eds). Acte du premier symposium international sur la panachure jaune du riz, 18-22 Septembre 1995. M'bé, Bouaké, (Côte d'Ivoire), WARDA, 109112.

Kouassi KN, N'Guessan P, Albar L, Fauquet MC et Brugidou C. 2005. Distribution and Characterization of Rice yellow mottle virus : a threat to African farmer. Plant Disease, 89(2): 125-133.

Roumen EC. 1993. Partial Resistance in Rice to Blast and How to Select for it : Latent Period to Leaf Blast in Rice and it Importance as a Component of Partial Resistance. Agricultural University Netherlands: Wageningen; 108.

Vales M, Yoboue N, Bouet A. 2001. Stratégie de sélection pour l'amélioration de la résistance au virus de la panachure jaune du riz à l'IDESSA en Côte d'Ivoire. In . La panachure jaune du riz : Importance économique, diagnostic et stratégie de gestion Abdoul Aziz Sy, Jacqueline Hughes and Alassane Diallo (eds). Acte $\mathrm{du}$ premier symposium international sur la panachure jaune du riz, 18-22 Septembre 1995, M’bé, Bouaké, (Côte d'Ivoire), WARDA, 188-196. 